\title{
Carrier relaxation and thermal activation of localized excitons in self-organized InAs multilayers grown on GaAs substrates
}

\author{
Z. Y. Xu, Z. D. Lu, X. P. Yang, Z. L. Yuan, B. Z. Zheng, and J. Z. Xu \\ National Laboratory for Superlattices \& Microstructures, Institute of Semiconductors, Academia Sinica, Beijing 100083, China \\ W. K. Ge, Y. Wang, J. Wang, and L. L. Chang \\ Department of Physics, Hong Kong University of Science \& Technology, Clear Water Bay, Kowloon, Hong Kong
}

(Received 8 April 1996)

\begin{abstract}
We have investigated the temperature dependence of photoluminescence (PL) properties of a number of self-organized InAs/GaAs heterostructures with InAs layer thickness ranging from 0.5 to $3 \mathrm{ML}$. The temperature dependence of InAs exciton emission and linewidth was found to display a significant difference when the InAs layer thickness is smaller or larger than the critical thickness around 1.7 ML. The fast redshift of PL energy and an anomalous decrease of linewidth with increasing temperature were observed and attributed to the efficient relaxation process of carriers in multilayer samples, resulting from the spread and penetration of the carrier wave functions in coupled InAs quantum dots. The measured thermal activation energies of different samples demonstrated that the InAs wetting layer may act as a barrier for the thermionic emission of carriers in high-quality InAs multilayers, while in InAs monolayers and submonolayers the carriers are required to overcome the GaAs barrier to escape thermally from the localized states. [S0163-1829(96)06440-5]
\end{abstract}

Spontaneous formation of three-dimensional (3D) islands during Stranski-Krastanov-like growth of highly strained InAs layers on GaAs substrates by molecular-beam epitaxy (MBE) has been proposed as a promising way for fabricating high-quality InAs quantum dots (QD's) in GaAs. ${ }^{1-6}$ When the thickness of an InAs layer is beyond a critical thickness of around $1.7 \mathrm{ML}$, the structure is usually composed of an InAs wetting layer and conelike InAs islands deposited on it. The density, size distribution, uniformity, and coverage of such InAs islands were found to be growth condition dependent, and have been investigated by TEM and atomic force microscopy (AFM) in recent years. ${ }^{7-9}$ The optical studies ${ }^{10-13}$ revealed its excellent radiative recombination, which usually gives a broadband with a reported full width at half maximum (FWHM) in the range of 50-130 meV. Recent work by Lubyshev et al. ${ }^{13}$ presented the unusual temperature dependence of exciton energy in InAs multilayer structures. An anomalous decrease of the FWHM was detected and explained in terms of the tunneling process between InAs dots.

In this paper, we studied the exciton relaxation and thermal activation in InAs multilayer structures via the analysis of $\mathrm{cw}$ photoluminescence (PL) data under different temperatures. It is found that the temperature dependence of the exciton energy and linewidth is significantly different from that obtained in InAs monolayers and submonolayers. The unusual temperature behavior in InAs multilayers is associated with the relaxation effect of carriers, resulting from the spread and penetration of the wave functions of carriers in coupled InAs QDs. In the study of the thermal activation process, we found that potential barriers for InAs excitons to escape thermally from the localized states are different for different structures. For high-quality InAs multilayers, the barrier could be the wetting layer, while in the case of monolayers or submonolayers the carriers are required to overcome the GaAs barrier in order to escape thermally from the localized states.

The samples studied here were grown by MBE on (100) GaAs substrates. A $0.5-\mu \mathrm{m}$ GaAs buffer layer was deposited first at $580{ }^{\circ} \mathrm{C}$, followed by a single InAs layer at $450{ }^{\circ} \mathrm{C}$. Then 20-nm GaAs was grown on the top at the same substrate temperature. The growth was monitored by reflection high-energy electron diffraction (RHEED), and the quantumdot nucleation was seen directly via the onset of a spotty RHEED pattern. The detailed growth description and their high quality as evidenced by TEM, scanning electron microscopy, and $\mathrm{x}$ ray will be published elsewhere. The PL measurement was performed in a variable-temperature (10$300 \mathrm{~K}$ ) closed-cycle cryostat under the excitation of a 514.5-nm line of an argon laser. The signal from samples was dispersed by a Jobin-Yven HR250 monochromator and detected by a cooled Ge detector.

Figure 1 shows low temperature PL spectra of InAs layers with different thicknesses of $1,1.5,1.8,2.5$, and $3 \mathrm{ML}$, respectively. All spectra are dominated by a strong luminescence related to the InAs exciton transitions. GaAs-related

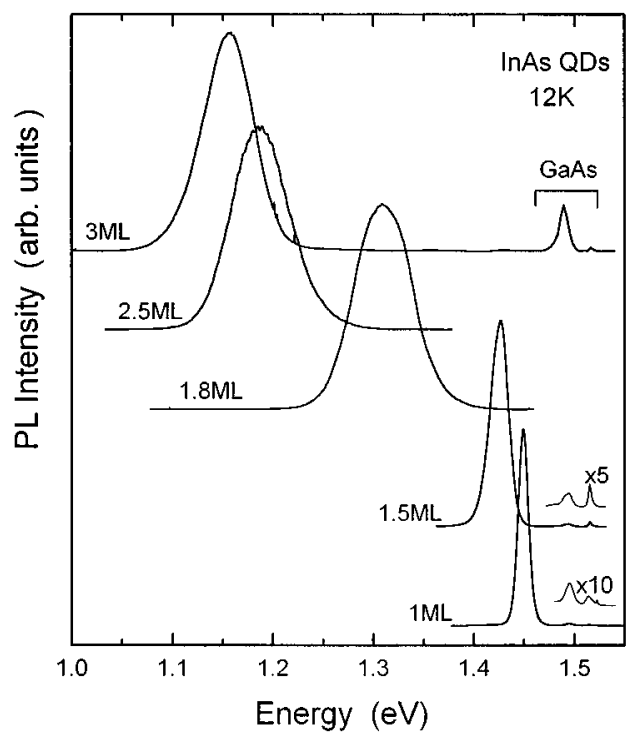

FIG. 1. Normalized PL spectra at $12 \mathrm{~K}$ of InAs layers with different thicknesses of $1,1.5,1.8,2.5$, and $3 \mathrm{ML}$, respectively. 
emissions (1.515 and $1.493 \mathrm{eV}$ ) are much weaker, even though GaAs has a much larger excitation volume. The observed high luminescence efficiency indicates an improved confinement of InAs excitons and a low defect density in the samples; the latter was further supported by the observed linear dependence of the integrated PL intensity on the excitation power. The high efficiency of the luminescence is also indicative of relieving restriction on the energy and momentum relaxation of carriers, known as the phonon bottleneck effect. ${ }^{14,15}$ The emission energy of the InAs layer is found to decrease monotonically with layer thickness, and could be estimated using the effective mass approximation. ${ }^{16}$ One remarkable feature in Fig. 1 is a sudden change of the FWHM of the InAs emission. When the InAs thickness is larger than $\sim 1.7 \mathrm{ML}$, the FWHM of the luminescence is dramatically increased, from $17 \mathrm{meV}$ for $1.5 \mathrm{ML}$ to $56 \mathrm{meV}$ for $2.5 \mathrm{ML}$. This can be understood in terms of spontaneous formation of InAs quantum dots and their size distribution. The size fluctuation (both in height and diameter) causes a distribution of the density of states over a wide energy range, resulting in a spectrally broad luminescence. Therefore, the sudden change of the FWHM gives direct optical evidence of the formation of InAs QD's. The size distribution was found to depend strongly on the growth conditions and structure parameters ${ }^{8,9}$ The control of the size distribution has been an open challenge for zero-dimensional quantum structures.

In Fig. 1, it is also noted that no luminescence related to the wetting layer was observed in PL, although the wetting layer was exposed in our TEM. The absence of the luminescence related to the wetting layer is most likely due to the fact that the carriers photogenerated in the wetting layer transfer to the adjacent InAs dots and then recombine there. Later we will show that this wetting layer may act as a potential barrier for the thermal quenching process of InAs carriers at high temperature.

Figure 2 presents the temperature dependence of the PL peak energy in different InAs samples. It is clear that the energy variation with temperature falls into two categories: when the InAs layer thickness is smaller than the critical thickness $(\sim 1.7 \mathrm{ML})$ the photon energy of the InAs layer follows the InAs band gap ${ }^{17}$ very closely (see the curves denoted 1 and 1.5 ML). This behavior is very similar to that observed in a $2 \mathrm{D}$ system. ${ }^{6}$ However, when the layer thickness is larger than $\sim 1.7$ ML (see, the curves denoted 2 and $2.5 \mathrm{ML}$ ), the rate of energy shift with temperature becomes much faster, and also shows sample dependence. For example, the rate for 2 ML (Ref. 18) is measured to be three times higher than the InAs band-gap variation, while the rate in the 2.5-ML sample is only 1.5 times higher. Consequently, in the temperature range from 12 to $160 \mathrm{~K}$, the peak energy of 2-ML InAs was redshifted by $65 \mathrm{meV}$ compared to 35 $\mathrm{meV}$ in the 2.5-ML sample. We believe that this unusual temperature dependence is a typical characteristic of selforganized InAs QD's, resulting from the enhanced carrier relaxation process related to the size distribution of quantum dots.

It is well recognized that the size fluctuation has been a problem in the fabrication of QD's. In the self-organized growth of InAs/GaAs layers, the uniformity of InAs dots depends strongly on growth conditions. ${ }^{8,9}$ On the other hand, when the InAs coverage is large enough, the dots may couple

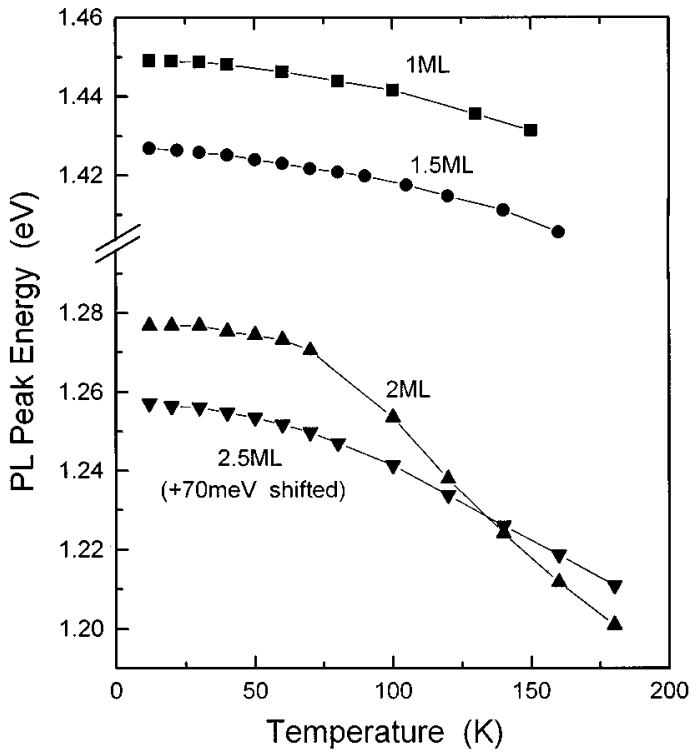

FIG. 2. Temperature dependence of the PL peak energy for InAs layers of $1,1.5,2$, and $2.5 \mathrm{ML}$, respectively. It is important to point out that the line shape of the PL spectra remains Gaussian in the temperature range we studied (now shown), indicating the size distribution behavior of QD's.

with each other. The whole system, therefore, can be regarded as a coupled system, and the wave function of carriers in an individual QD can penetrate into adjacent dots, relieving or partially relieving the phonon relaxation bottleneck by increasing the number of states related to carrier relaxation. When the temperature increases, the coupling and relaxation effects will be enhanced due to increased electronphonon interaction. As a result, photogenerated carriers transfer and relax into energetically low-lying states, giving rise to the fast redshift of the exciton energy. As the FWHM can be taken as a measure of the size distribution of QD's in a sample, a faster redshift of energy would be then expected in a sample with wider linewidth. In our experiments, the FWHM of the 2-ML sample is $75 \mathrm{meV}$, compared to 56 $\mathrm{meV}$ in the 2.5-ML InAs. This explains the observed faster redshift of PL energy in the 2-ML sample.

An efficient relaxation process is also expected to reduce the linewidth at higher temperatures. Shown in Fig. 3 is the FWHM of the PL as a function of temperature for the samples of Fig. 2. Again, a significant difference can be seen for InAs layer thicknesses larger or smaller than the critical thickness. When the InAs thickness is smaller than $\sim 1.7$ ML, the FWHM increases monotonously with temperature. The increase of the linewidth with temperature in the InAs monolayer was observed before, and attributed to electronphonon scattering. ${ }^{6,19,20}$ However, in the case of InAs multilayers, an anormalous decrease of the linewidth with increasing temperature was observed in the temperature range from 12 to $130 \mathrm{~K}$ (see the curve denoted as $2 \mathrm{ML}$ ). This can be explained as follows: The broad linewidth at low temperature is mainly determined by the distribution of the localenergy minimum of QD's caused by size fluctuation of InAs islands. When temperature increases, the thermalized carriers can relax over a long distance and find a lower local-energy minimum, resulting in a shrinkage of the linewidth. When 


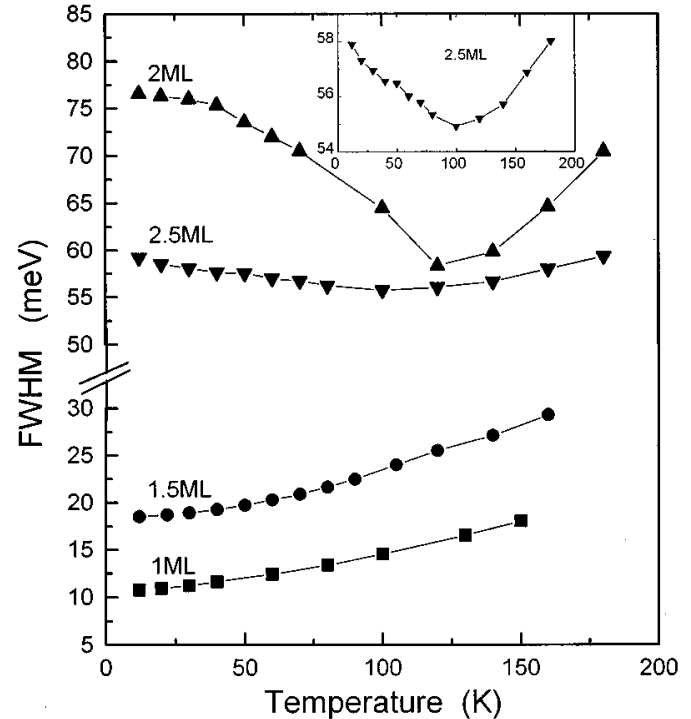

FIG. 3. FWHM of PL spectra as a function of temperature for the samples in Fig. 2. The inset is the results of the 2.5-ML sample with an enlarged vertical scale, showing clearly the same common behavior as the 2-ML sample.

the temperature further increases (above $130 \mathrm{~K}$ ), both the electron-phonon scattering and thermal distribution become important. Consequently, the FWHM increases with temperature. We note that the above-mentioned behavior is common for both 2 and 2.5-ML samples, although the rate of variation could be different. We believe that this difference is partly associated to the uniformity of sample.

The temperature dependence of the wavelength-integrated intensity of InAs luminescence is displayed in Fig. 4. Using the dependence of $I=C \exp \left(E_{A} / k T\right),{ }^{21-23}$ we extract the corresponding thermal activation energies from the hightemperature region of the figure, where the observed thermal quenching of the PL is mainly related to the escape process of the dissociated excitons (electron-hole pairs) into the corresponding barriers.

Thermal activation energies of 27, 64, 110, and $105 \mathrm{meV}$ were estimated for 0.5-, 1-, 2-, and 2.5-ML InAs, respectively. Note that the values of 27 and $64 \mathrm{meV}$ are very close to 28 and $67 \mathrm{meV}$, the energy differences between $\mathrm{GaAs}$ band-gap and InAs exciton emissions in the 0.5- and 1-ML samples, respectively. While the values of 110 and $105 \mathrm{meV}$ are comparable to 106 and $120 \mathrm{meV}$, the energy differences between the PL emissions of InAs QD's and the wetting layers $^{24}$ for the 2- and 2.5-ML InAs samples, respectively. Our results suggest that, for InAs monolayers or submonolayers the barrier is GaAs, while for high-quality InAs multilayers the wetting layer may serve as a barrier for the thermionic emission of carriers. Finally it is important to point

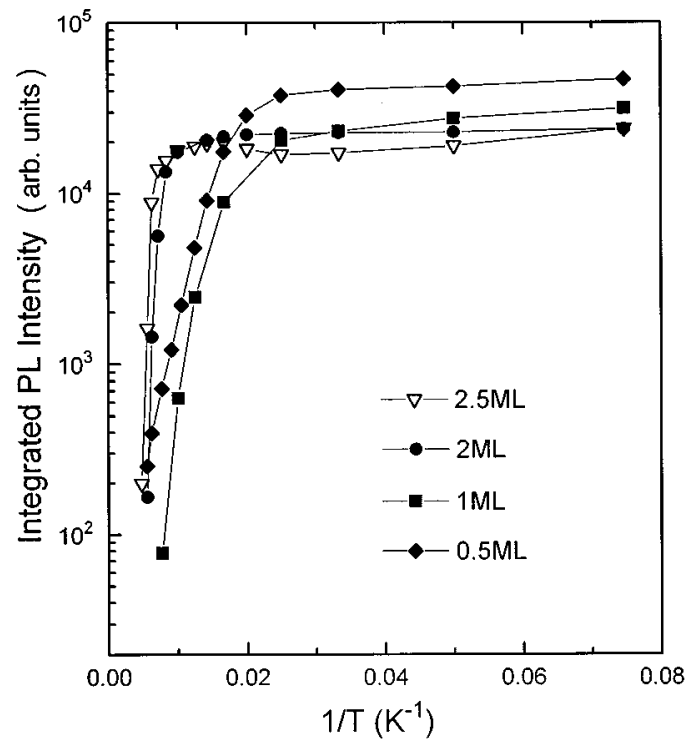

FIG. 4. Temperature dependence of the wavelength-integrated intensity of InAs layers.

out that the thermal activation energy measured from the Arrhenius plot is not always comparable to the values related to GaAs or InAs wetting-layer barriers. Any defects and dislocations in the material will provide nonradiative channels to quench the luminescence, and consequently reduce the thermal activation energy. This was observed in our thick (3 and $6 \mathrm{ML}$ ) InAs samples.

In conclusion, by studying the temperature dependence of the PL we are able to study the exciton relaxation process and thermal activation in self-organized InAs quantum dots. A sudden increase of the PL linewidth at an InAs thickness around $\sim 1.7 \mathrm{ML}$ indicated a spontaneous formation of InAs QD's and their size distribution. The enhanced relaxation of photogenerated carriers was evidenced by the experimentally observed fast redshift of PL energy, and an anomalous decrease of linewidth with increasing temperature. In studying the thermal activation process we found that the potential barriers for InAs excitons to escape thermally from localized states are different when the InAs thickness is larger or smaller than the critical thickness. For high-quality InAs multilayers, the barrier could be the wetting layer, while in the case of monolayers or submonolayers the carriers are required to overcome the GaAs barrier in order to escape thermally from the localized states.

This work was supported by the National Natural Science Foundation of China and the State Key Program for Basic Research, and Grant MFG 94/95. SC03 at Hong Kong University of Sciences and Technology.
${ }^{1}$ J. M. Marzin and J. M. Gerard, Phys. Rev. Lett. 62, 272 (1989).

${ }^{2}$ D. Leonard, M. Krishnamurth, C. M. Reaves, S. P. Denbaars, and P. M. Petroff, Appl. Phys. Lett. 63, 3203 (1993).

${ }^{3}$ J. M. Moison, F. Houzay, F. Barthe, L. Leprince, E. Andre, and O. Vatel, Appl. Phys. Lett. 64, 196 (1994).
${ }^{4}$ K. Ploog and O. Brandt, Semicond. Sci. Technol. 8, S229 (1993).

${ }^{5}$ P. D. Wang, N. N. Ledentsov, C. M. Sotomayern Torres, P. S. Kop'ev, and V. M. Ustinov, Appl. Phys. Lett. 64, 1526 (1994).

${ }^{6}$ Z. L. Yuan, Z. Y. Xu, B. Z. Zheng, J. Z. Xu, S. S. Li, Weikun Ge, 
Yuqi Wang, Jiannong Wang, and L. L. Chang, Phys. Rev. B (to be published).

${ }^{7}$ D. Leonard, K. Pond, and P. M. Petroff, Phys. Rev. B 50, 11687 (1994).

${ }^{8}$ H. Klitabayashi and T. Waho, J. Cryst. Growth 150, 152 (1995).

${ }^{9}$ G. S. Solomon, J. A. Trezza, and J. S. Harris, Jr., Appl. Phys. Lett. 66, 3161 (1995).

${ }^{10}$ J. Y. Marzin, J. M. Gerard, A. Izrael, D. Barrier, and G. Bastard, Phys. Rev. Lett. 73, 716 (1994).

${ }^{11}$ J. M. Gerard, J. B. Genin, J. Lefebvre, J. M. Moison, N. Lebouche, and F. Barthe, J. Cryst. Growth 150, 351 (1995).

${ }^{12}$ A. Tuckeuchi, Y. Natkata, S. Muto, Y. Sugiyaya, T. Inata, and N. Yokoyama, Jpn. J. Appl. Phys. 34, L405 (1995).

${ }^{13}$ D. I. Lubyshev, P. P. Gonalez-Borrero, E. Marega, Jr., E. Petitprez, N. La Scala, Jr., and P. Basmaji, Appl. Phys. Lett. 68, 205 (1996)

${ }^{14}$ U. Bockelmann and G. Bastard, Phys. Rev. B 42, 8947 (1990).

${ }^{15}$ H. Benisty, C. M. Sotomayor Torres, and C. Weisbuch, Phys. Rev. B 44, 10945 (1991).

${ }^{16}$ S. S. Li, J. B. Xia, Z. L. Yuan, Z. Y. Xu, W. K. Ge, X. R. Wang,
Y. Wang, J. Wang, and L. L. Chang, Phys. Rev. B (to be published).

${ }^{17}$ J. Pankove, Optical Processes in Semiconductors (Dover, New York, 1975), p. 27.

${ }^{18}$ The 2-ML InAs sample was grown under different conditions. The PL spectrum of the sample was shown to be wider than those in the other InAs multilayers.

${ }^{19}$ Y. J. Chen, E. S. Koteles, J. Lee, J. Y. Chi, and B. S. Elman, Proc. SPIE 792, 168 (1987).

${ }^{20}$ D. Gammon, S. Rudin, T. L. Reincke, D. S. Katzer, and C. S. Kyono, Phys. Rev. B 51, 16785 (1995).

${ }^{21}$ E. W. Williams and H. B. Bebb, J. Phys. Chem. Solids 30, 1289 (1969).

${ }^{22}$ G. Bacher, C. Hartmann, H. Schweizer, T. Held, G. Mahler, and H. Nickel, Phys. Rev. B 47, 9545 (1993).

${ }^{23}$ J. D. Lambkin, D. J. Dunstan, K. P. Homewood, L. K. Howaed, and M. T. Emeny, Appl. Phys. Lett. 57, 1986 (1990).

${ }^{24}$ The photon energy of the wetting layer is taken to be $1.307 \mathrm{eV}$, the value directly obtained from a sample of 1.7 ML thick grown under the same conditions. 